\title{
Concomitant challenge of a very large cervical fibroid with bilateral ovarian masses
}

\author{
Ashok R. Anand, Dhruv Pravin Gohil*
}

Department of Obstetrics \& Gynaecology, Grant Government Medical College and Sir JJ Group of Hospitals, Mumbai, Maharashtra, India

Received: 18 September 2014

Accepted: 10 October 2014

*Correspondence:

Dr. Dhruv Pravin Gohil,

E-mail: detartsurf@gmail.com

Copyright: (C) the author(s), publisher and licensee Medip Academy. This is an open-access article distributed under the terms of the Creative Commons Attribution Non-Commercial License, which permits unrestricted non-commercial use, distribution, and reproduction in any medium, provided the original work is properly cited.

\begin{abstract}
Occurrence of cervical fibroids is rare. Seldom have they grown huge. Cases with associated ovarian masses are rare and frequently misdiagnosed. A 44 year old, postmenopausal female, presented with abdominal distension and pain. Radiology was suggestive of broad ligament fibroid with ovarian masses. On laparotomy she was found to have huge ovarian masses with a huge cervical fibroid. Bilateral mass excision done followed by cervical myomectomy followed by hysterectomy was done. Cervical fibroids may give rise to greater surgical difficulty by virtue of relative inaccessibility and close proximity to bladder and ureter. Occurrence of bilateral huge ovarian masses with a huge cervical fibroid is very rare. The management of such cases requires great surgical skill.
\end{abstract}

Keywords: Large, Cervical, Fibroid, Ovarian, Mass

\section{INTRODUCTION}

Uterine fibroids are widespread. Most of the patients are asymptomatic or without any menstrual irregularities. Symptomatic fibroids in women bear an enormous disease burden and have a negative impact on the quality of life. It is not uncommon to incidentally diagnose uterine fibroids of considerable size. Many women with large uterine fibroids are symptomless.

Although presence of concomitant endometriosis is seen many times in fibroid cases, large ovarian masses with large sized fibroids, especially extra uterine like cervical fibroids are very rare.

Fibroids are benign monoclonal tumors believed to be of myometrial origin. They develop in women in the reproductive age group and their growth is predominantly driven by reproductive hormones. Most commonly used system to classify fibroids in relation to uterine fibroids is the location that is intramural, subserosal, sub mucosal.
STEPW classification includes fibroid size, location and depth of invasion. Intramural fibroids do not disrupt the endometrial cavity and have less than $50 \%$ protrusion beyond the serosal surface. Subserosal fibroids are those with more than $50 \%$ protrusion beyond the serosal surface. Submucosal fibroids are the ones which protrude into the endometrial cavity \& distort the uterine cavity \& have been classified into three types - type 0 , type $1 \&$ type 2 based on the ESHRE/ESGE classification.

Whereas the basic underlying physiology may be identical the triggering mechanism of fibroid development at particular fibroid may vary among other fibroids in single uterus.

Cervical fibroids can change the shape of cervix and may push the uterus upwards and can obstruct the cervix. Large cervical fibroids distort the pelvic anatomy especially of the urological system (ureters and bladder). We need an expert hand to operate these cases. Here we are presenting one such case. 


\section{CASE REPORT}

A 44 year old, postmenopausal patient, 5 full term home deliveries followed by a caesarean section 13 years back came with complains of abdominal distension and pain since 2 months. She had no previous menstrual complaints.

On examination, vitals were stable. On per abdomen examination she had a 30 weeks size mass arising from pelvis, which was mobile, firm, non-tender, lower pole, could not be palpated.

On per speculum examination cervix was flushed with vagina. On per vaginum examination the pelvic mass was felt. All routine investigations were normal. CA-125 was normal.

Ultrasound and CT scan showed bilateral large ovarian masses with a large adnexal mass suggestive of a ? Broad ligament fibroid with hydro ureter and minimal ascites. Doppler showed increased resistance. Preoperatively ureteric stenting was tried but could not be done.

On opening the abdomen, there were bilateral huge ovarian masses of approximate size $15 \times 10 \times 8 \mathrm{~cm}$ size, both twisted on its own axis with minimal fluid in abdomen and no secondary deposits, and a huge cervical fibroid of $20 \times 15 \times 10 \mathrm{~cm}$ size. Uterus was normal size. Bilateral ovarian masses were excised \& sent for frozen section. After ligating the uterine vessels, cervical myomectomy was done after pushing the bladder down and separating posterior peritoneum from the uterus \& keeping the ureters under vision. Frozen section report showed benign nature of the masses, hence hysterectomy was done.

After confirming the urinary tract integrity and hemostasis abdomen was closed. Patient's postoperative course was uneventful.

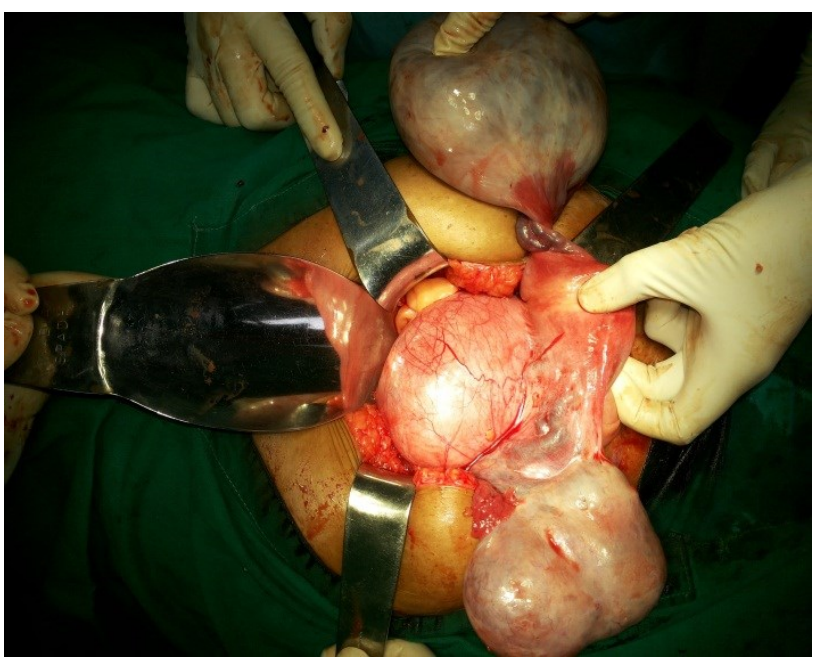

Figure 1: Intraoperative picture showing the large cervical fibroid with twisted ovarian masses.

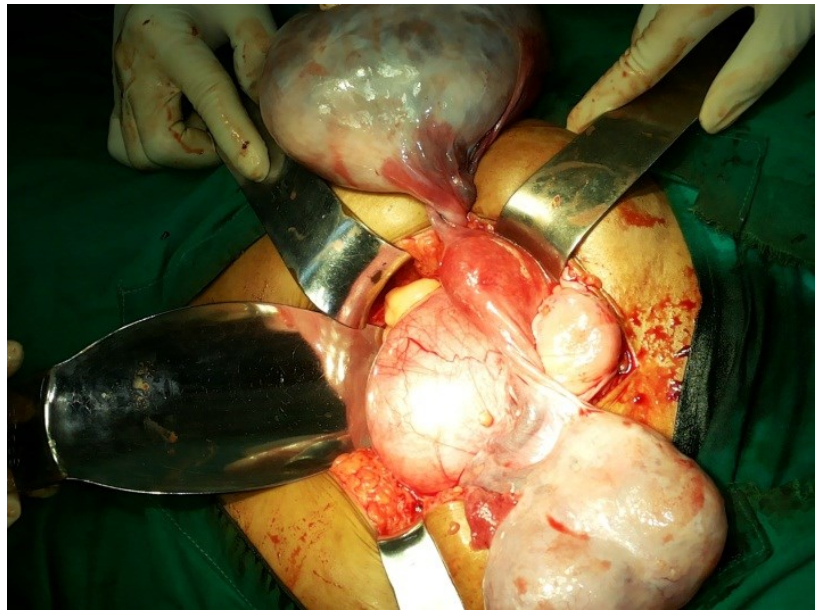

Figure 2: Intraoperative picture showing the masses.

\section{DISCUSSION}

Cervical fibroids are uncommon. These fibroids grossly and histopathalogically are identical to those found in the uterus. Fibroids with excessive growth may cause pressure symptoms. Treatment of cervical fibroid is either hysterectomy or myomectomy. ${ }^{1}$ They may give rise to greater surgical difficulty by virtue of relative inaccessibility and close proximity to bladder and ureter. ${ }^{2}$ Very large cervical fibroids are always a surgical challenge. Bilateral ovarian masses pose a yet another challenge. Presence of cervical fibroid with ovarian masses is rare in literature.

May be hormonal correlation between the two could be the reason but considering the rarity of its concurrence and very common occurrence of fibroids, rule out this possibility. Anatomical distortion occurs in cervical fibroids especially of the ureters and bladder. It is not always possible to do stenting of ureters and also stenting causes mucosal damage to ureters and also surgical intervention to remove the stent later. Hence the best way of preventing ureteric injury is to identify the ureter and keep under observation whenever you are clamping or tying any pelvic structure or pedicle. Keeping close to the uterus helps. Opening posterior peritoneum and separating it from the uterus before cervical myomectomy, opening the anterior fold of peritoneum carefully, pushing the bladder down and retracting especially at the angle will also reduce bladder/ureteric injury.

\section{CONCLUSION}

Although fibroids are very common, surgery is not always easy or without any complications. Extra-uterine fibroids occur infrequently, although they are histologically benign, may mimic malignant tumors at imaging, and may present a diagnostic challenge. It depends on the size, location and number of fibroids. Preoperative ureteric stenting helps but not always. Preoperative embolization in cervical fibroid may not be 
of great help. So only way one can reduce complications is by carefully dissecting bladder and ureters and keeping them under vision during surgery. Ovarian masses should be dealt as a separate pathology rather than considering them a part of it. But occurrence of bilateral large ovarian masses with a very large cervical fibroid is very rare. The management of such cases requires great surgical skill.

\section{Abbreviations}

- STEPW classification - Site, Topography, Extension of the base, Penetration, Wall (Lateral), A classification based on the above parameters

- ESHRE - the European Society of Human Reproduction and Embryology and ESGE - the European Society for Gynaecological Endoscopy

- CA 125 - Carbohydrate Antigen 125 (Normal values between $0-35 \mathrm{U} / \mathrm{ml}$ )
Funding: No funding sources Conflict of interest: None declared Ethical approval: Not required

\section{REFERENCES}

1. Basnet N, Banerjee B, Badani U, Tiwari A, Raina A, Pokhari $\mathrm{H}$, et al. An unusual presentation of huge cervical fibroid. Khatmandu Univ Med J. 2005;3(2):173-4.

2. Kshirsagar SN, Laddad MM. Unusual presentation of cervical fibroid: two case reports. Int J Gynaecol Plast Surg. 2011;3(1):38-9.

DOI: $10.5455 / 2320-1770 . i j r \operatorname{cog} 20150248$

Cite this article as: Anand AR, Gohil DP. Concomitant challenge of a very large cervical fibroid with bilateral ovarian masses. Int J Reprod Contracept Obstet Gynecol $2015 ; 4: 252-4$. 